\title{
Secular trends in proximal femoral fracture, Oxford record linkage study area and England 1968-86
}

\author{
J Grimley Evans, Valerie Seagroatt, M J Goldacre
}

\begin{abstract}
Objective-To study hospital admission rates for firactures of the proximal femur over a period when incidence is reported to have increased, compensating for known lack of precision in coding, excluding nonemergency admissions and transfers, and modelling for age, period, and cohort effects. Design-Validation of coding of a sample of hospital admissions followed by study of two sets of routinely collected statistical abstracts of hospital records; graphical analysis and statistical modelling were used to search for period and cohort effects.
\end{abstract}

Setting-Oxfordshire and west Berkshire in 1968-86, covered by the Oxford record linkage study (ORLS), and England in 1968-85, covered by the hospital inpatient enquiry (HIPE). The ORLS and HIPE datasets are almost independent (ORLS contributed about $1.8 \%$ of the HIPE data).

Subjects-Records of patients aged 65 and over.

Outcome measures-Admission rates for fractured neck of femur and fracture of other and unspecified parts of femur (N820 and N821), and evidence of period and cohort effects.

Results-The validation study indicated that it was important to combine the codes 820 and 821 in this age group. Admission rates increased over the period studied in both HIPE and ORLS datasets. In HIPE the pattern was of two plateaux separated by a period of rapid rise in the late 1970s. In the ORLS data there was a more steady rise. Statistical analysis showed significant period and cohort effects but much of this was attributable to the component of the model common to both period and cohort effects (termed "drift").

Conclusions-The finding that admission rates increased in both datasets, combining relevant codings and restricting analysis to emergency admissions, strongly suggests that the rise was real. At least part of the period effect in the HIPE data, however, might be attributable to a sampling artefact. The cohort effect in incidence rates of femoral fracture has not been previously shown and would be compatible with a number of aetiological hypotheses.

(f Epidemiol Community Health 1997;51:424-429)

Proximal femoral fracture (PFF) comprises fractures of the cervical and trochanteric regions of the femur. It is one of the three main "osteoporotic fractures" of later life (the others being of the distal forearm and the vertebrae), although it can occur at younger ages following severe trauma. In later life it is thought to differ significantly in aetiology from fractures of the femoral shaft, with which it may be confounded in routine health service statistics owing to coding errors.

An increase in the incidence of PFF has been reported from a number of countries over recent decades but the timing and the pattern of the increase has varied. The explanation remains elusive but suggestions abound including patterns of physical exercise, ${ }^{1}$ dietary changes including calcium intake, ${ }^{2}$ smoking, change in body habitus, ${ }^{4}$ and variation in ultraviolet exposure determined by atmospheric ozone concentrations. ${ }^{5}$ Some of these factors would be expected to increase rates mainly through differences between successive cohorts, others more through period effects affecting different age cohorts simultaneously. Analysis of the secular trends in PFF incidence to ascertain the contributions of period and cohort effects is therefore potentially useful.

Most of the reports of increasing incidence have been based on routinely collected hospital admission data which are subject to a range of diagnostic and other errors. We report here trends for the period 1968-86 in data from the Oxford record linkage study (ORLS) after evaluation of samples of patients' records from the beginning, middle, and end of the study period to assess diagnostic error. We also present data for similar diagnostic groupings from national hospital inpatient enquiry (HIPE) data. HIPE was intended to be based on a random 1 in 10 sample of all inpatient episodes in England. In practice the sample was not invariably random or 1 in 10 . Changes in the design and collection of NHS statistics since 1985 have rendered hospital admission data no longer reliable for epidemiological use.

ORLS has collected and linked information, including hospital admission data, for defined populations around Oxford since the mid 1960 s. $^{6}$ A preliminary study of ORLS data indicated an increase in the incidence of PFF between the single years 1966 and $1979^{7}$ and an increase between years 1954-58 and 1983 has also been shown in an independent set of data from the Oxford region. ${ }^{1}$ Increases in incidence rates of PFF based on HIPE data have also been reported. ${ }^{89}$

Methods

VALIDATION STUDY

Previous studies have shown that significant errors can occur in the coding of femoral frac- 
Table 1 Numbers of cases in the Oxford record linkage study (ORLS) data coded correctly as ICD N820 or N821, coded incorrectly as one rather then the other, and cases not identified or traced. There were no cases coded as N820 or N821 which were not femoral fracture

\begin{tabular}{llrrr}
\hline \multirow{2}{*}{ Year } & Coding status & \multicolumn{2}{c}{ ORLS code } & \\
\cline { 3 - 5 } & & 820 & 821 & Total \\
\hline $1968-70$ & Coded correctly & 59 & 15 & 74 \\
& Coded incorrectly & 6 & 31 & 37 \\
& Wrong identification & 2 & 16 & 24 \\
$1976-78$ & Untraceable & 8 & 13 & 21 \\
& Coded correctly & 60 & 38 & 98 \\
& Wrong code & 5 & 27 & 32 \\
& Wrong identification & 3 & 1 & 4 \\
$1984-86$ & Untraceable & 7 & 9 & 16 \\
& Coded correctly & 58 & 35 & 93 \\
& Wrong code & 3 & 25 & 28 \\
Total & Wrong identification & 3 & 1 & 4 \\
& Untraceable & 11 & 14 & 25 \\
& Coded correctly & 177 & 88 & 265 \\
& Wrong code & 14 & 83 & 97 \\
Total & Wrong identification & 8 & 18 & 26 \\
& Untraceable & 26 & 36 & 62 \\
& & 225 & 225 & 450 \\
\hline
\end{tabular}

tures in routine hospital data. Rees ${ }^{10}$ showed that the main error is for PFF to be allocated to N821 "fracture of other and unspecified parts of femur" rather than to N820 "fracture of neck of femur". (In practice this error usually occurs because of an imprecise written description of the injury on discharge documents rather than through mistakes in subsequent coding.) We therefore wished to validate the ORLS data by identifying any change over the study period in the accuracy of the coding that might produce spurious variation in apparent incidence. In order to distinguish a trend from sporadic fluctuation we sampled the beginning, middle, and end of the total study sequence. For each of the three year periods 1968-70, 1976-78, and 1984-86 a random sample of 75 records was identified from ORLS data for each of the two diagnostic codes 820 and 821 . (Records in ORLS were coded to the 8th revision of the International Classification of Diseases from 1968 to 1978 and to the 9th revision from 1979 to 1986 . Records were sampled for people aged 65 or over who had been treated in a City of Oxford hospital.)

In addition to diagnostic code, hospital record number, age, sex, date of birth, and date of admission were extracted from the data. With the permission of the relevant consultants, the hospital notes were then sought and the diagnosis re-coded on the basis of radiographic or operative reports. Cases were then allocated to one of the four categories of "fractured femur correct coding", "fractured femur incorrect coding" (N820 should have been N821 or vice versa), "identification error" (hospital notes traced on the basis of hospital number found to relate to wrong patient on the basis of age or sex discrepancy), and "notes untraceable". There were no cases found in which the diagnosis was not fractured femur.

The results of this validation exercise are presented in table 1 . The comparatively high number of irretrievable records due to wrong identification numbers in the first of the three periods studied is probably due to merging of the numbering systems of the records departments of the hospitals involved, one of which has since closed. Assuming no bias due to the untraced cases, $32 \%(83 / 260)$ of the true cases of PFF had been coded as fractures of other or unspecified parts of the femur. Of the 171 cases that had been coded to N821, 83 (48.5\%) were cases of PFF that should have been coded to N820. Incorrect coding of other femoral fractures as PFF was rarer; only 14 (7.3\%) of the 191 cases coded to N820 should have been N821. There was no evidence of any trend in coding practice over the period but the magnitude of the error was such that we deemed it appropriate for the two categories to be combined in an examination of secular changes. For individuals aged 65 and over, codes of $\mathrm{N} 820$ outnumbered those of $\mathrm{N} 821$ by about 5.7 to 1 in ORIS data for the years 1979-85. On the basis of our validation exercise therefore we estimate that in hospital discharge data at that time around $86 \%$ of the two categories combined were true PFF.

The full ORLS dataset was searched for cases admitted in each calendar year from 1967-86 and the age and sex of all patients admitted with a diagnosis coded to N820 or N821 were identified. Analysis was restricted to cases coded as being immediate admissions in order to exclude inter-hospital transfers. Cases with codes indicating admission for late effects of fractures (in 820.9 and 821.9 from 1968 to 1978 and 905.3 and 905.4 from 1979 to 1986 ) were excluded. "Populations at risk" for each calendar year were calculated from census data for 1971 and 1981 with interpolations from OPCS estimates. The number of districts contributing to the ORLS database increased over the period but our analysis was restricted to data from the two districts providing data for the full period of our study.

Data were also extracted from HIPE tapes for immediate admissions of patients with a diagnosis coded as N820 or N821 over the period 1968 to 1985 after which data ceased to be available. Codes for late effects of fractures were excluded. Hospital admission rates were calculated for each year of HIPE using appropriate populations and published multiplication factors for the precise fraction of records in the HIPE sample.

\section{STATISTICAL METHODS}

The effects of age, period, and cohort were estimated by fitting log-linear models as described by Clayton and Schifflers. ${ }^{112}$ Unfortunately, period and cohort effects are not independent. If the period effect is a trend in which the ratio of age-specific rates is constant across age groups and across time periods then the age-period model is equivalent to the agecohort model in which the ratio between adjacent birth cohorts is constant. Clayton and Schifflers termed this regular trend "drift". We have followed their recommendation and assessed the significance of cohort and period effects after adjustment for the drift component so that each can be tested for significance independently of the common component. This was done by adding period effects to the age + drift model and noting the improvement in fit by assessing whether the change in de- 

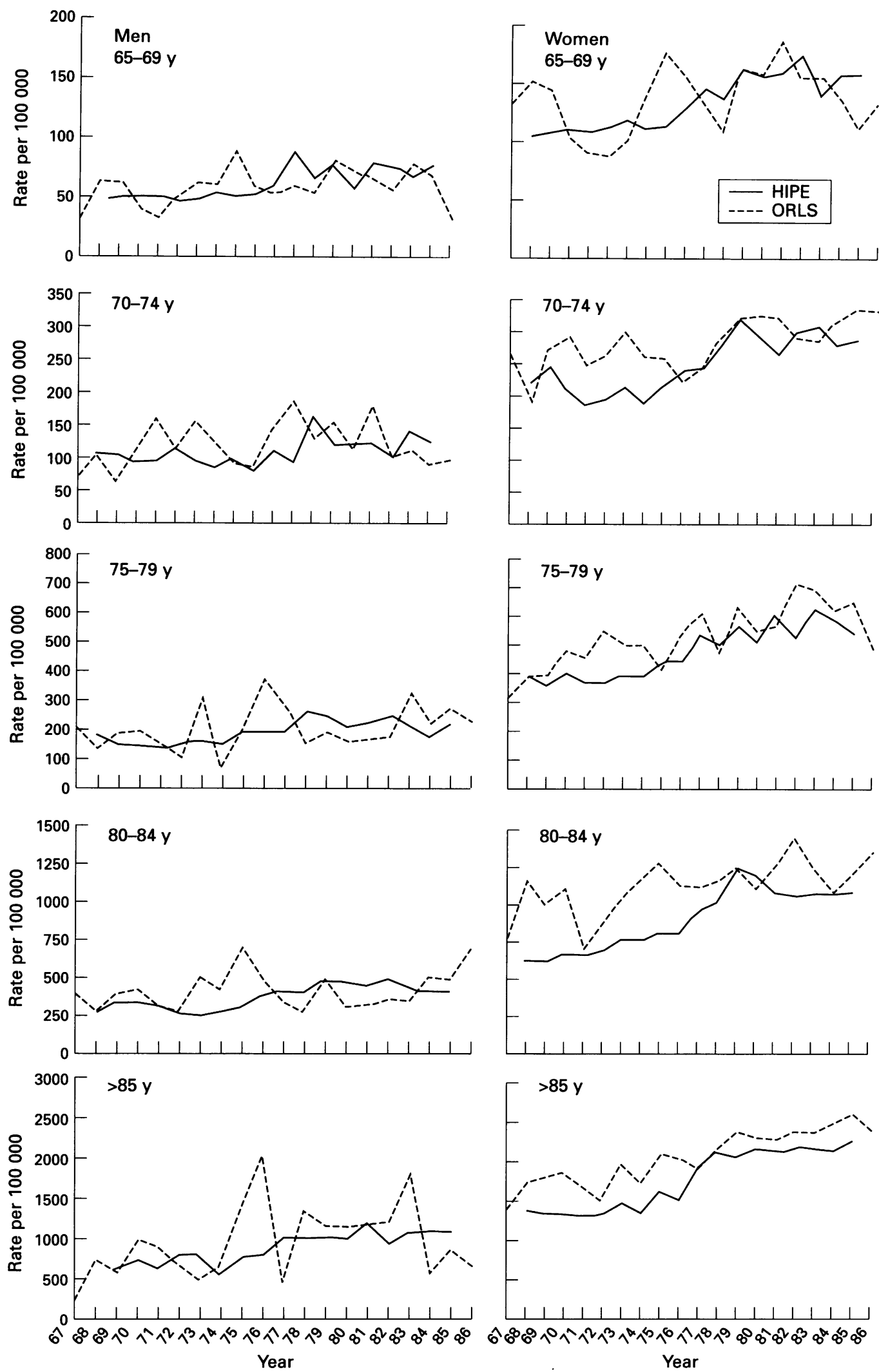

Figure 1 Sex specific and age specific rates of immediate hospital admissions for fractured femur: hospital inpatient enquiry (HIPE) data (1968-85) and Oxford record linkage study (ORLS) data (1967-86).

viance (which approximates to a $\chi^{2}$ statistic) was significant. The significance of the cohort effect was assessed in a similar way. We also added the period effects to the age + cohort model and the cohort effects to the age + period model and assessed the significance of the resulting reductions in deviance.

\section{Results}

Trends in sex specific and age specific rates of $\Omega$ PFF in the population aged 65 and over in the HIPE and ORLS data are shown in figure 1. Figure 2 shows the trends for men and women in the two sets of data standardised by the direct method in five-year age groups (up to 


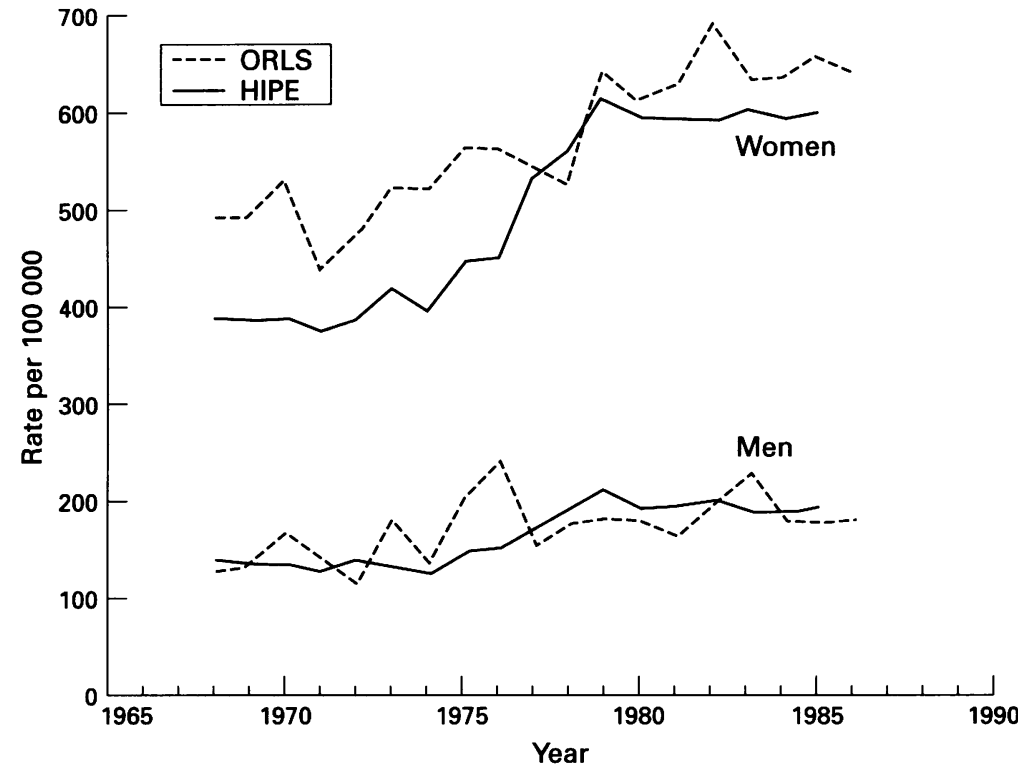

Figure 2 Hospital admission rates for fractured femur from Oxford record linkage study (ORLS) and hospital inpatient enquiry (HIPE) data standardised on to 1981 populations by five year age groups from 65 to 85 and over.

85 and over) on to the 1981 populations. Both datasets show an increase in rates over the study period for all age groups and both sexes. For the HIPE data the increase was most marked during the period 1976-79, while for the ORLS data there was a more continuous trend. For women, rates in the ORLS districts were higher than national estimates from HIPE in the first half of the study period but were similar in the latter half.

For the HIPE data the period and cohort effects were each highly significant (respectively $\chi^{2}=1548: \mathrm{df}=3$ and $\left.\chi^{2}=1403: \mathrm{df}=7\right)$ but most of these effects could be attributed to the common component of drift $\left(\chi^{2}=1339\right.$ : $\mathrm{df}=$
1). Allowing for drift, the period effects were still significant $\left(\chi^{2}=209: \mathrm{df}=2: \mathrm{p}<0.01\right)$ as were the cohort effects but to a less extent $\left(\chi^{2}=\right.$ 64: $d f=6: p<0.01)$. Further, the addition of the period effects to the sex + age + cohort model showed marked improvement in fit $\left(\chi^{2}=175\right.$ : $\mathrm{df}=2: \mathrm{p}<0.01$ ) while the addition of cohort effects to the sex + age + period model showed less of an improvement $\left(\chi^{2}=30: \mathrm{df}=6\right.$ : $\mathrm{p}<0.01)$. These findings indicate that for the HIPE data, after taking account of the common drift factor, the changes could be attributed more to a period than a cohort effect.

The ORLS data also showed a highly significant drift component $\left(\chi^{2}=110: \mathrm{df}=1\right.$ : $\mathrm{p}<0.01)$. Unlike the HIPE data neither the period nor the cohort effects were significant after subtracting the drift component (respectively $\left(\chi^{2}=2.3: \mathrm{df}=2:\right.$ and $\chi^{2}=9.6: \mathrm{df}=6$ : $\mathrm{p}>0.05)$. However, the ORLS data set contained only about a fifth as many patients with PFF as did the HIPE data (9000 and 46000 respectively). Thus the statistical tests were not as powerful in the ORLS as in the HIPE data. However, the parameter estimates for the sex + age, sex + age + period and sex+age + cohort models did not differ much between the two datasets (table 2). The fitted parameters for the period effects in HIPE showed a jump between 1972-76 and 1977-81 while those for ORLS showed a more steady increase. This confirms the visual impression from figure 2 . In contrast the HIPE parameters for the cohort effects showed a steady increase while the ORLS appeared to flatten out over the three most recent cohorts.

Figure 3 presents the HIPE data for women plotted for overlapping birth cohorts. This gives a visual impression of an interaction of cohort and period effects in incidence.

Table 2 Parameters of the age, sex, period, and cohort models fitted to the hospital inpatient enquiry (HIPE) data (1968-85) and the Oxford record linkage study (ORLS) data (1967-86)

\begin{tabular}{|c|c|c|c|c|c|c|c|c|}
\hline & \multicolumn{2}{|l|}{ No } & \multicolumn{2}{|c|}{$A g e+s e x$} & \multicolumn{2}{|c|}{ Age + sex + period } & \multicolumn{2}{|c|}{ Age + sex + cohort } \\
\hline & HIPE & ORLS & HIPE & ORLS & HIPE & ORLS & HIPE & ORLS \\
\hline \multicolumn{9}{|l|}{ Men: age (y) } \\
\hline $65-69$ & 1180 & 188 & .30 & .27 & .31 & .27 & .23 & .24 \\
\hline $70-74$ & 1585 & 288 & .54 & .55 & .55 & .55 & .47 & .57 \\
\hline 75-79* & 1777 & 324 & 1 & & & 1 & & 1 \\
\hline $80-84$ & 1745 & 351 & 1.97 & 2.09 & 1.99 & 2.11 & 2.32 & 2.37 \\
\hline 85 & 1985 & 379 & 4.60 & 4.62 & 4.64 & 4.67 & 6.41 & 5.87 \\
\hline \multicolumn{9}{|l|}{ Women: age (y) } \\
\hline 65-69 & 3259 & 521 & .27 & .25 & .27 & .26 & .21 & .22 \\
\hline $70-74$ & 5324 & 944 & .51 & .52 & .51 & .52 & .44 & .48 \\
\hline $75-79^{*}$ & 7895 & 1367 & 1 & 1 & 1 & 1 & 1 & 1 \\
\hline $80-84$ & 9468 & 1892 & 1.91 & 2.12 & 1.91 & 2.13 & 2.24 & 2.39 \\
\hline 85 & 12228 & 2561 & 3.71 & 3.89 & 3.69 & 3.88 & 5.12 & 4.85 \\
\hline \multicolumn{9}{|l|}{ Sex } \\
\hline Men* & 8272 & 1530 & 1 & 1 & 1 & 1 & 1 & 1 \\
\hline \multirow{2}{*}{\multicolumn{9}{|c|}{ Year of fracture }} \\
\hline & & & & & & & & \\
\hline $1968-71^{*}$ & 7507 & 1517 & - & - & 1 & 1 & - & - \\
\hline $1972-76$ & 10865 & 1978 & - & - & 1.08 & 1.15 & - & - \\
\hline $1977-87$ & 15251 & 2404 & - & - & 1.49 & 1.27 & - & - \\
\hline $1982-85$ & 12831 & 2916 & - & - & 1.53 & 1.37 & - & - \\
\hline \multicolumn{9}{|l|}{ Year of birth } \\
\hline $1860-84$ & 2052 & 480 & - & - & - & - & .58 & .69 \\
\hline $1885-89$ & 4990 & 1035 & - & - & - & - & .65 & .80 \\
\hline $1890-94$ & 8868 & 1596 & - & - & - & - & .85 & .91 \\
\hline 1895-99* & 11403 & 2188 & - & - & - & - & 1 & 1 \\
\hline $1900-04$ & 8829 & 1620 & - & - & - & - & 1.12 & 1.09 \\
\hline $1905-09$ & 6029 & 1137 & - & - & - & - & 1.29 & 1.27 \\
\hline 1910-14 & 3205 & 578 & - & - & - & - & 1.51 & 1.28 \\
\hline $1915-19$ & 1010 & 181 & - & - & - & - & 1.66 & 1.24 \\
\hline
\end{tabular}

* Reference value

† Sex ratio given for age group 75-79 years 


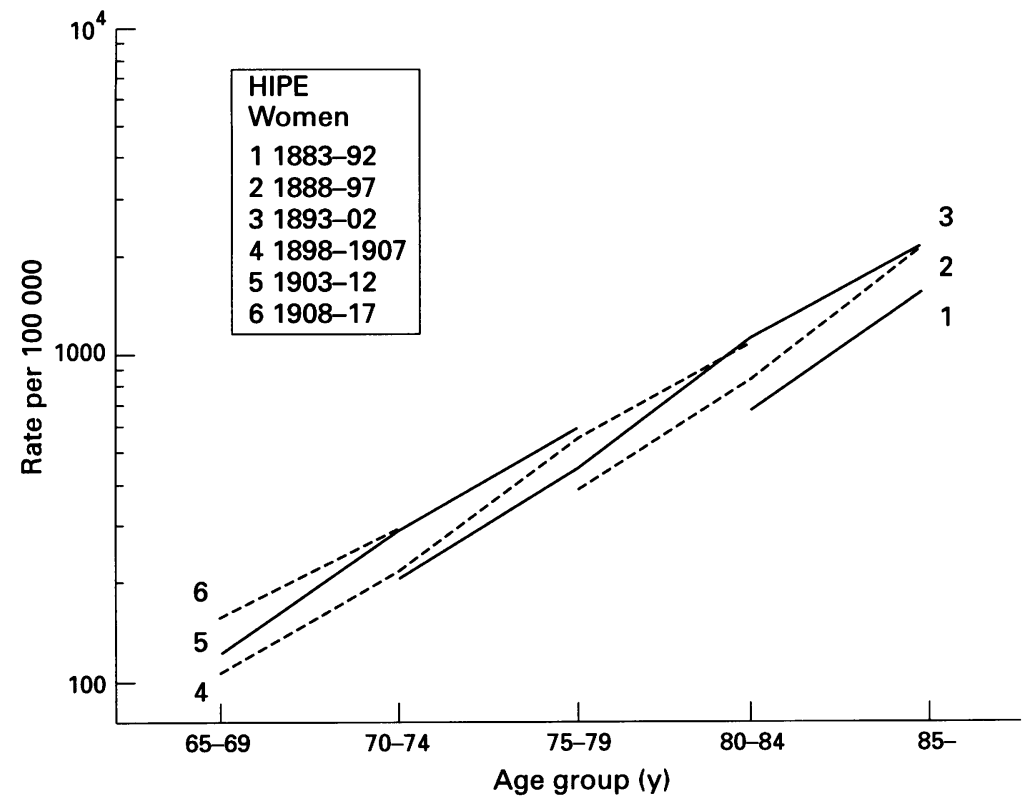

Figure 3 Hospital admission rates for women with a fractured femur plotted for overlapping birth cohorts; hospital inpatient enquiry (HIPE) data.

\section{Discussion}

HIPE and ORLS are almost independent datasets. ORLS included all inpatient episodes in two health districts in the time period covered by this analysis. HIPE consisted of an approximately 1 in 10 sample of all inpatient episodes in England; the two ORLS districts would have constituted about $1.8 \%$ of the HIPE data.

The grouping of N820 and N821 codes used in our analysis will have removed the main known source of recording error in PFF data. The restriction to immediate admissions and exclusion of admission due to late effects of fracture in our analysis would also prevent any inflation of rates due to transfer between orthopaedic and geriatric units. A previous publication based on HIPE data for PFF (N820 alone) and including non-emergency admissions and transfers, showed that rates for all ages over 45 had increased by between $50 \%$ and $60 \%$ in both sexes from 1968 to 1978 but had been more or less constant thereafter. ${ }^{9}$ Our analysis for the diagnostically selected HIPE data, combining codes N820 and N821 and restricted to direct admissions excluding late effects, differs in finding that rates did not increase until around 1974. HIPE data presented by Fenton Lewis ${ }^{8}$ show that over the period 1968 to 1974 the numbers of admissions coded to N821 were falling, although those coded to N820 were rising faster. While this might represent true changes in the epidemiology of fractures of the proximal and more distal femur it could also indicate a trend of improvement in the precision of coding. We did not find any such trend in our validation study of Oxford data but there is evidence ${ }^{10}$ that the accuracy of coding may in the past have been higher on average in teaching centres. We also need to explain the notably higher incidence rates, particularly in women, in ORLS compared with HIPE prior to the mid 1970s. Coding errors in HIPE seem an unlikely explanation since our analysis combined codes N820 and N821 and a significant loss in HIPE of cases of PFF into codes other than N821 seems implausible. We speculate that the lower rates in HIPE in the early part of the period studied was due to selective under-representation of admissions for fractured femur in the sample forming the basis of the HIPE dataset. For example, a study in Newcastle upon Tyne in 1976 (J Grimley Evans unpublished observations) suggested that patients transferred (with their clinical notes) for rehabilitation or long-stay care to peripheral hospitals following PFF were under-sampled in HIPE. This particular problem would be more likely to affect women than men given the difference between the sexes in rates of widowhood and consequent lack of support at home. Some of the increase in HIPE rates in the late 1970s that brought them up to the levels of the ORLS dataset (derived from 100\% recording of hospital admissions) could then be due to improvement in sampling practice. Such a change would be expected to generate a period effect in apparent incidence.

In formal statistical analysis of the HIPE data significant cohort and period effects were found after exclusion of the "drift" factor ie change which is not attributable specifically to period or cohort effects. ${ }^{11}$ Part at least of the period effect might be attributable to the improvement in HIPE sampling of PFF admissions suggested above. In the smaller dataset from ORLS, neither period nor cohort effects alone was statistically significant after exclusion of the shared "drift" component, although the estimated parameters were broadly similar in the two datasets. The ORIS data suggest that rates may have continued to rise, albeit slowly, in the 1980 s, but this trend is not seen in HIPE. The cohort component was apparent (subject to the statistical limitations already noted) in both datasets for births from 1860-64 to 1905-9 but although continuing to increase up to births in 1915-19 in the HIPE data, no further increase was apparent in ORLS. HIPE data reflect the national situation in England while the ORLS data set relate to a limited district in central England. There may be regional differences in the pattern of secular increase in PFF incidence. In a series of studies from Newcastle upon Tyne ${ }^{3}$ rates for women were increasing through the 1970 s but were stable for men. Rates in Oxford and Newcastle, which had probably differed during the period 1966 to $1976,{ }^{7}$ were identical by the early $\mathrm{O}$ 1980s. ${ }^{3}$

Clayton and Schifflers ${ }^{11} 12$ point out that there can be no unique mathematical solution for a three factor model (age, period, cohort) using a two dimension dataset (age, time) unless there are biological reasons for setting one or $\mathbb{D}$ more of the parameters. For some diseases there may be a biological basis for predicting the relationship between incidence and age. This is not so for PFF but in the great majority $\mathbb{D}$ of data its incidence has a broadly exponential relationship with age which showed a notable parallelism in independent cross sectional data $\delta$ from two different times and places in England. ${ }^{7}$ This is unhelpful in interpreting our 
findings since, as table 2 indicates, both the ageperiod and age-cohort models produce broadly exponential age slopes in the two sexes.

Our analysis suggests that the incidence of PFF has been increasing in a broadly similar pattern in men and women. We need to find explanations for a powerful period effect over 1974 to 1980 and an increasing risk in successive cohorts of the English population born after 1860 .

There are three groups of factors involved in the genesis of PFF. ${ }^{13}$ These comprise: factors associated with weakness of bones; factors associated with the falls which generate most fractures; and protective factors in falls. The last includes active responses by the falling subject and more passive factors such as the direction of falling and the padding effects of subcutaneous fat, clothing, and floor coverings. Much of the speculation surrounding the rise in incidence of PFF has focused on an assumption that the underlying causes act through an increase in the prevalence of osteoporosis. The only direct evidence of a secular increase in this condition comes from a comparison of skeletons from an 18 th century burial chamber with data from the present day. ${ }^{14}$ It is not known at what stage in the last 200 years the increase in osteoporosis took place. Given the complex aetiology and pathogenesis of PFF it might be more realistic to speculate that trends in incidence might arise not so much through changes in any one single factor but as a result of interactions between changes in a number of factors perhaps in all three of the pathogenic groups identified above.

One factor to be considered is body height which is known to have increased in successive birth cohorts from the end of the last century in Britain and which is positively associated with risk of PFF in epidemiological studies. In a middle aged Norwegian population the relative risk associated with increase in height of $0.1 \mathrm{~m}$ was 1.58 (95\% confidence interval (CI) $1.18,2.12)$ in women and $2.19(95 \% \mathrm{CI}$ $1.46,3.28$ ) in men. ${ }^{15}$ The rate of increase in height in the British population over the early years of this century was of the order of one centimetre per decade, at least in men. ${ }^{16}$ Increase in height may therefore have contributed to the observed cohort increase in PFF incidence but is an insufficient explanation given the magnitude of the differences between cohorts. ${ }^{17}$ Other possible contributors to cohort effects in femoral fracture incidence include dietary calcium intake in childhood, ${ }^{2}$ cigarette smoking, ${ }^{318}$ and levels of physical activity. ${ }^{19}$ The possible importance of low vitamin D intake in the genesis of osteoporosis and PFF has recently been explored in observational and interventive studies. ${ }^{2021}$ Secular changes in vitamin $\mathrm{D}$ intake or in exposure to ultraviolet radiation ${ }^{5}$ could produce cohort or period effects or both. Deficiency of vitamin D activity sufficient to produce osteomalacia seems to have become rarer rather than more common in recent decades in patients with $\mathrm{PFF}^{22}$

Much of the period effect detected in the HIPE data involves the abrupt stepwise in- crease from 1974-79. This is probably attributable, at least in part, to some form of artefact as it is difficult to conceive of an environmental factor that could produce so dramatic a change in several age groups and both sexes simultaneously and over so short a period.

In conclusion, the problem of "drift" and the consequent impossibility of identifying changes in incidence specifically with period or cohort effects is apparent in both the datasets we have analysed. After removing the component of "drift" there is evidence in both the datasets of period and cohort effects in the recent increase in the incidence of PFF in England, although these effects were only statistically significant in the larger set. The period effect is stronger statistically but in the ORLS data may have been partly due to a sampling artefact. Although less prominent statistically the cohort effect is biologically more plausible.

The work reported here was supported in part by a grant from the Nuffield Provincial Hospitals Trust. Thanks are due to Margaret Savory and Maureen Courtenay-Thompson for extracting the data for the validation study. The Unit of HealthCare Epidemiology is funded by the Anglia and Oxford Regional Office of the National Health Service Executive. We thank the Office of Population Censuses and Surveys for the Hospital Inpatient Enquiry data.

1 Boyce WJ, Vessey MP. Rising incidence of fracture of the proximal femur. Lancet 1985;i:150-51.

2 Heaney RP. Effect of calcium on skeletal development, bone loss, and risk of fracture. Am $\mathcal{F}$ Med 1991;91 (Suppl 5B): loss, and risk.

3 Grimley Evans J. Incidence of proximal femoral fracture. Lancet 1985;i:925-26.

4 Reid IR, Chin K, Evans MC, Jones JG. Relation between increase in length of hip axis in older women between 1950 s and 1990s and increase in age specific rates of hip fracture. $B M \mathcal{F}$ 1994;309:508-9.

5 Leach JF, Beadle PC, Pingstone AR. Effect of ozone variation on disease in Great Britain: femoral neck fracture. Aviation Space and Environmental Medicine 1978;49:1014-18.

6 Acheson ED, Grimley Evans J. The Oxford record linkage study: a review of the method and some preliminary results. Proc Roy Soc Med 1964;57:269-74.

7 Rees JL. Secular changes in the incidence of proximal femoral fracture in Oxfordshire: a preliminary report. Community Med 1982;5:100-3.

8 Fenton Lewis A. Fracture of neck of the femur: changing incidence. $B M \mathcal{F}$ 1981;283:1217-20.

9 Spector TD, Cooper C, Fenton Lewis A. Trends in admission for hip fracture in England and Wales, 1968-85.
mector TD, $B M \mathcal{1}$ 1990;300:1173-74

10 Rees JL. Accuracy of hospital activity analysis data in estimating the incidence of proximal femoral fracture. $B M \mathcal{F}$ 1982;284: 1856-57.

11 Clayton D, Schifflers E. Models for temporal variation in cancer rates. I. Age-period and age-cohort models. Stat Med 1987;6:449-67.

12 Clayton D, Schifflers E. Models for temporal variation in cancer rates. II. Age-period-cohort models. Stat Med 1987; 6:469-81.

13 Grimley Evans J. Epidemiology of proximal femoral fracture. In: Isaacs B ed. Recent advances in geriatric medicine 2. Edinburgh: Churchill Livingstone 1982;201-14

14 Lees B, Molleson T, Arnett TR, Stevenson JC. Differences in proximal femur bone density over two centuries. Lancet in proximal femur

15 Meyer HE, Tverdal A, Falch JA. Risk factors for hip fracture in middle-aged Norwegian women and men. Am $\mathcal{J}$ Epidemiol 1993;137:1203-11.

16 Benn RT. Some mathematical properties of weight for height indices used as a measure of obesity. $\mathrm{Br} \mathcal{F}$ Prev Soc Med 1971;25:42-50.

17 Grimley Evans J. Proximal femoral fracture. In: Ebrahim $\mathrm{S}$, Kalache A, eds. Epidemiology in old age. London, BMJ Publishing Group, 1996;300-10.

18 Anonymous. Anti-oestrogenic effect of cigarette smoking. Editorial. Lancet 1986;ii: 1433 .

19 Boyce WJ, Vessey MP. Habitual physical inertia and other factors in relation to fracture of the proximal femur. Age Ageing 1988;17:319-27.

20 Dawson-Hughes B, Dallal GE, Krall EA, Harris S, Sokoll LJ, Falconer G. Effect of vitamin D supplementation on wintertime and overall bone loss in healthy postmenopausal women. Ann Int Med 1991;115:505-12.

21 Chapuy MC, Arlot ME, Delmas PD, Meunier PJ. Effect of calcium and cholecalciferol treatment for three years on hip fractures in elderly women. $B M F 1994 ; 308: 1081-82$.

22 Compston JE, Vedi S, Croucher PI. Low prevalence of osteomalacia in elderly patients with hip fracture. Age Ageing 1991;20:132-34. 